\title{
Lyapunov stability analysis of a system coupled to a hyperbolic PDE with potential *
}

\author{
Mohammed Safi( ${ }^{(1)}$ Alexandre Seuret ${ }^{(1)}$ Lucie Baudouin ${ }^{(1)}$
}

\begin{abstract}
This work deals with a stability problem for a system coupling an ordinary differential equation to a linear vectorial hyperbolic transport equation with a potential term. Using the Lyapunov methodology, a novel approach for stability of the coupled ODE-PDE system is developed. This methodology leads to a linear matrix inequality criteria while exploiting Bessel inequality and Legendre polynomials. To demonstrate the efficiency of this technique, the obtained criteria are applied on academic example.
\end{abstract}

\section{INTRODUCTION}

The analysis and control of infinite dimensional systems represent an emerging topic in automatic control that attracts an increasing number of scientific works [1], [5], [12]. The main reason is related to the fact that many physical systems are described by PDEs such as traffic flow control [11], tokamak [18], or temperature control in intelligent buildings [3] among many others. When considering stability analysis and control of such models, one approach consists in working on a finite dimensional discretized model to avoid some of the difficulties of the original system. But a system of very large dimension could be inevitable, leading to some specific difficulties. In some other situations, such discretized approximations may not be relevant since the main features of the infinite dimensional dynamics could be disregarded and, for instance, spill-over phenomena may occur (i.e. the designed controller may stabilize the finite dimensional approximated system but excite the very first neglected dynamics, a situation that happens in vibration's control [8]). As a matter of fact, the stability or control study of infinite dimensional systems involving partial differential equations (PDE) represents a challenging area of research.

A large literature have been published on stability and control of this class of system, and we will not try to be exhaustive here. One instance could be given with [13] where a boundary control is developed for a first-order hyperbolic PDE using backstepping method. One can also find the study of a system coupling hyperbolic first-order PDEs in [7] which designs a controller requiring a single control input to stabilize the whole system. Recently, the coupling between PDEs and ordinary differential equations (ODEs) attracted

* The paper was partially supported by the ANR projects LimICoS and SCIDIS. Corresponding author . Fax +33-561336411.

(1) LAAS-CNRS, Université de Toulouse, CNRS, Toulouse, France. some specific attention regarding stability and control issues, as in [17] which develops a stability study of a coupled ODE-PDE system introducing a perturbation into the hyperbolic PDE, [10] designing a feedback laws to stabilize a fan-shaped network given by this class of system and [4] treating a fluidodynamic model for traffic flow. An alternative approach was presented in $[2],[14],[15]$, where stability conditions of linear ODEPDE systems expressed in terms of expandable Linear Matrix Inequalities (LMI) results from the application of the Bessel-Legendre inequality [16].

The present article aims at extending the stability study of [15] in the situation where the transport equation is also affected by a linear, space independent potential term. Actually, the introduction of a potential in the transport PDE yields a more complicated analysis and requires a dedicated attention and treatment that we will present here.

The paper is organized as follows. The next section formulates the problem and provides some preliminaries. Section 3 presents the main features of the proposed Lyapunov approach. Afterwards, Section 4 exposes the main results of the paper on the stability analysis of the coupled system. Finally, the last section draws some conclusions and perspectives.

Notations: $\mathbb{R}^{n}$ is the $n$-dimensional Euclidean space with vector norm $|\cdot|_{n}$ and $\mathbb{N}$ is the set of positive integers. The set of the diagonal positive matrices of dimension $m$ is $\mathbb{D}_{m}^{+}$and the set of real matrices of dimension $n \times m$ is given by $\mathbb{R}^{n \times m}$. $I_{n} \in \mathbb{R}^{n \times n}$ is the identity matrix, $0_{n, m}$ the null matrix, and $\left[\begin{array}{ll}A & B \\ \star & C\end{array}\right]$ replaces the symmetric matrix $\left[\begin{array}{cc}A & B \\ B^{\top} & C\end{array}\right]$. We denote $\mathbb{S}^{n} \subset \mathbb{R}^{n \times n}$ (respectively $\mathbb{S}_{+}^{n}$ ) the set of symmetric (resp. symmetric positive definite) matrices and $\operatorname{diag}(A, B)$ is a bloc diagonal matrix equal to $\left[\begin{array}{ll}A & 0 \\ 0 & B\end{array}\right]$. For any square matrix $A$, we define $\operatorname{He}(A)=$ $A+A^{\top}$. Finally, $L^{2}\left(0,1 ; \mathbb{R}^{n}\right)$ represents the space of square integrable functions over the interval $] 0,1[\subset \mathbb{R}$ with values in $\mathbb{R}^{n}$ and the partial derivative in time and space are denoted $\partial_{t}$ and $\partial_{x}$, while the classical derivative are $\dot{X}=\frac{d}{d t} X$ and $\mathcal{L}^{\prime}=\frac{d}{d x} \mathcal{L}$.

\section{Problem formulation}

\section{A. System data}

In this work, we consider the following system coupling the ODE with the finite variable $X(t) \in \mathbb{R}^{n}$, to a PDE transport equation with the infinite dimension variable 


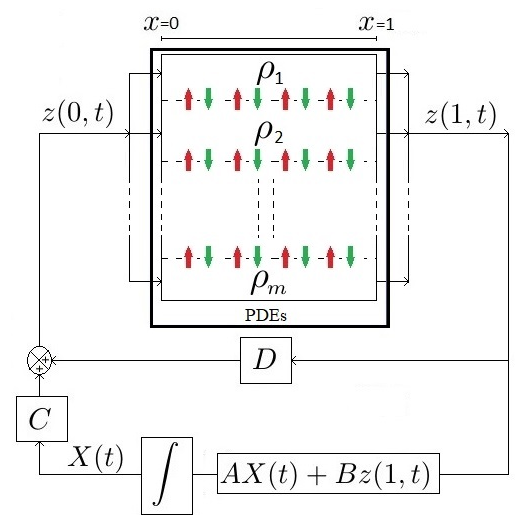

Fig. 1: Illustration of system (1).

$z(x, t) \in \mathbb{R}^{m}$

$\begin{cases}\dot{X}(t)=A X(t)+B z(1, t) & t>0, \\ \partial_{t} z(x, t)+\Lambda \partial_{x} z(x, t)+F z(x, t)=0, & x \in(0,1), t>0, \\ z(0, t)=C X(t)+D z(1, t), & t>0 .\end{cases}$

The vector $(X(t), z(x, t))$ forms the complete state of this coupled system and satisfies the initial condition $(X(0), z(., 0)) \in \mathbb{R}^{n} \times L^{2}\left(0,1 ; \mathbb{R}^{m}\right)$. The matrices $A, B$, $C$ and $D$ are constant with appropriate dimensions. The other parameters will be defined in the next paragraph. In system (1), the ODE is coupled to a transport PDE of matrix speed $\Lambda=\operatorname{diag}\left(\rho_{i} I_{m_{i}}\right)_{\{i=1 \ldots p\}} \in \mathbb{D}_{+}^{m}$, where $\rho_{i}>0$ is the applied speed to $m_{i}$ components of the variable $z(x, t)$, and the matrix $F \in \mathbb{R}^{m}$ in the potential term consists to interconnect different PDEs only in the undifferentiated terms. The total number of components $m$ is given by the sum $m=\sum_{i=1}^{p} m_{i}$.

\section{B. Description and applications}

To clarify the considered problem, system (1) can be illustrated as in Figure 1, where we can note that the potential term creates many canals between transport PDEs and allows all components of $z(x, t)$ to act in different equations.

PDE systems have been widely used in many important applications in the modeling and control of physical networks : gas pipe networks [10], road traffic [4] and hydraulic [5]. In the considered coupled model of this work, the ODE can be taken as a control for the perturbation introduced by PDE, and which is an undesirable effect in the coupled ODE-PDE system.

Remark 1: Since the transport matrix $\Lambda$ is diagonal, we can remark that, if the matrix $F$ is diagonal too, system (1) reduces to a set of independent scalar hyperbolic equations (see [14]). We consider the transport equation in system (1), with the change of variable $y(x, t)=e^{-\Lambda F x} z(x, t)$ allows proving this result.

In this problem, we consider that the matrix $F$ in system (1) can be any matrix in $\mathbb{R}^{m \times m}$. The effect of the lower order term, $F z(x, t)$, is to cause growth, decay, or oscillations in the solution, but it does not alter the primary feature of the propagation of the solution along the characteristics.

Remark 2: Note that all velocities $\rho_{i}$ of the transport speed matrix $\Lambda$ are positive. To treat the negative situation $\rho_{i}<0$ for some $i<p$ with the same formulation of the coupled system (1), we only need to apply the following change of state spatial variable $x^{\prime}=1-x$.

\section{Solution of the coupled system (1)}

The existence of solutions to system (1) can be easily proven using Theorem A.6 in [1]. Hence, for every $z^{0} \in$ $L^{2}\left(0,1 ; \mathbb{R}^{m}\right)$ and $X^{0} \in \mathbb{R}^{n}$, the Cauchy problem (1) has a unique solution. Moreover, there exist $K>0$ and $\delta>0$ such that the solution $(z(x, t), X(t))$ of system (1) satisfies :

$$
\|X(t)\|+\|z(t)\|_{L^{2}\left(0,1 ; \mathbb{R}^{m}\right)} \leq K e^{\delta t} .
$$

This well-posedness result suggests the choice of the following total energy of the system $E(X(t), z(t))=$ $|X(t)|_{n}^{2}+\|z(t)\|_{L^{2}\left(0,1 ; \mathbb{R}^{m}\right)}^{2}$. This energy will be expressed simply by $E(t)$.

To study the stability of this solution, we will follow the Lyapunov method, which is based on appropriate selection of a functional, suitable for the coupled system (1).

\section{Construction of Lyapunov functional for SYSTEM (1)}

In this paper, we aim at leading a stability study for system (1) following the same method as in [15]. The Lyapunov functional is usually constructed from the complete state of the system (1) which is $(X(t), z(x, t))$. Our functional contains four terms. A first quadratic term, generally chosen for the vector $X(t)$ of the ODE and an integral term for the infinite dimensional variable $z(x, t)$. The other two terms are dedicated to the coupling between the ODE and the PDE. This Lyapunov functional is given by

$$
\begin{gathered}
V_{N}(X(t), z(t))=\left[\begin{array}{c}
X(t) \\
Z_{N}(t)
\end{array}\right]^{\top}\left[\begin{array}{cc}
P & Q_{N} \\
Q_{N}^{\top} & T_{N}
\end{array}\right]\left[\begin{array}{c}
X(t) \\
Z_{N}(t)
\end{array}\right] \\
+\int_{0}^{1} z^{\top}(x, t)(S+(1-x) R) z(x, t) d x,
\end{gathered}
$$

where

$$
\begin{aligned}
& P \in \mathbb{S}_{+}^{n}, \quad S, R \in \mathbb{S}_{+}^{m}, \\
& Q_{N}=\left[\begin{array}{lll}
Q(0) & \ldots & Q(N)
\end{array}\right] \quad \in \mathbb{R}^{n, m(N+1)}, \\
& T_{N}=[T(i, j)]_{i, j=0 . . N} \\
& \in \mathbb{S}^{m(N+1)}
\end{aligned}
$$

and

$$
Z_{N}(t)=\left[\begin{array}{c}
\int_{0}^{1} z(x, t) \mathcal{L}_{0}(x) d x \\
\vdots \\
\int_{0}^{1} z(x, t) \mathcal{L}_{N}(x) d x
\end{array}\right] \in \mathbb{R}^{m(N+1)}
$$


is the projection of the $m$ components of the infinite dimensional state $z(x, t)$ over the $N+1$ first shifted Legendre polynomials (see for instance [6]). The family $\left\{\mathcal{L}_{k}\right\}_{k \in \mathbb{N}}$ of those polynomials forms an orthogonal basis of $L^{2}(0,1 ; \mathbb{R})$ and $\forall j, k \in \mathbb{N}$ the scalar product is $\int_{0}^{1} \mathcal{L}_{j}(x) \mathcal{L}_{k}(x) d x=\frac{1}{2 k+1} \delta_{j k}$, where $\delta_{j k}$ represents Kronecker's coefficient, equal to 1 if $j=k$ and 0 otherwise. We denote the corresponding norm of this inner scalar product $\left\|\mathcal{L}_{k}\right\|=1 / \sqrt{2 k+1}$.

We use Legendre polynomials to take advantage of their properties presented in his derivative and boundary values. The boundary values for all $k$ in $\mathbb{N}$ are $\mathcal{L}_{k}(0)=$ $(-1)^{k}$ and $\mathcal{L}_{k}(1)=1$, and the space derivative expression is

$$
\mathcal{L}_{k}^{\prime}(x)= \begin{cases}0, & k=0 \\ \sum_{j=0}^{k-1} \ell_{k j} \mathcal{L}_{j}(x), & k \geq 1\end{cases}
$$

where

$$
\ell_{k j}= \begin{cases}(2 j+1)\left(1-(-1)^{k+j}\right), & \text { if } j \leq k-1, \\ 0, & \text { if } j \geq k .\end{cases}
$$

Those properties will simplify our computing hereafter. The Lyapunov functional must satisfy properties of positivity and differentiability by respecting the following inequalities

$$
\begin{gathered}
\varepsilon_{1} E(t) \leq V_{N}(X(t), z(t)) \leq \varepsilon_{2} E(t), \\
\dot{V}_{N}(X(t), z(t)) \leq-\varepsilon_{3} E(t)
\end{gathered}
$$

for some positive scalars $\varepsilon_{1}, \varepsilon_{2}$ and $\varepsilon_{3}$.

We note in (7) that we have to compute the time derivative of the Lyapunov functional to verify the inequality, and since the Lyapunov functional defined in (2) is in function of the projection vector $Z_{N}$ (see (3)), we need to compute the time derivative of this last. This derivative is provided in the following lemma.

Property 1: Consider $z \in C\left(\mathbb{R}_{+} ; L^{2}\left(0,1 ; \mathbb{R}^{m}\right)\right)$ satisfying the transport equation in system (1). The time derivative of the projections vector $Z_{N}$ is given by :

$$
\begin{gathered}
\dot{Z}_{N}(t)=\left(L_{N}(\Lambda)-\mathcal{F}_{N}\right) Z_{N}(t)+\left(\mathbb{1}_{N}^{*}(\Lambda) D-\mathbb{1}_{N}\right) z(1, t) \\
+\mathbb{1}_{N}^{*}(\Lambda) C X(t)
\end{gathered}
$$

where

$$
\begin{aligned}
\mathbb{1}_{N}(\Lambda) & =\left[\begin{array}{lll}
\Lambda & \ldots & \Lambda
\end{array}\right]^{\top} \in \mathbb{R}^{m(N+1), m} \\
\mathbb{1}_{N}^{*}(\Lambda) & =\left[\begin{array}{llll}
\Lambda & -\Lambda & \ldots & (-1)^{N} \Lambda
\end{array}\right]^{\top} \in \mathbb{R}^{m(N+1), m},(8) \\
\mathcal{F}_{N} & =\operatorname{diag}(F, F, \ldots, F) \in \mathbb{R}^{m(N+1), m(N+1)} \\
L_{N}(\Lambda) & =\left[\begin{array}{ll}
\left.\ell_{k j} \Lambda\right]_{j, k=0 . . N} \in \mathbb{R}^{m(N+1), m(N+1)}
\end{array}\right.
\end{aligned}
$$

the $\ell_{k j}$ being defined in (5).

Proof: We can easily compute the time derivative of $Z_{N}(t)$ using the transport equation in (1), integration by parts and properties of the Legendre polynomials. We obtain the following expression:

$$
\begin{aligned}
& \frac{d}{d t} \int_{0}^{1} z(x, t) \mathcal{L}_{k}(x) d x=\int_{0}^{1} \partial_{t} z(x, t) \mathcal{L}_{k}(x) d x \\
& =-\int_{0}^{1}\left(\Lambda \partial_{x} z(x, t)+F z(x, t)\right) \mathcal{L}_{k}(x) d x \\
& =-\left[\Lambda z(x, t) \mathcal{L}_{k}(x)\right]_{0}^{1}+\int_{0}^{1} \Lambda z(x, t) \mathcal{L}_{k}^{\prime}(x) d x \\
& -F \int_{0}^{1} z(x, t) \mathcal{L}_{k}(x) d x \\
& =-\Lambda z(1, t)+(-1)^{k} \Lambda z(0, t)-F\left(Z_{N}(t)\right)_{k} \\
& +\sum_{j=0}^{\max [0, k-1]} \ell_{k j} \Lambda \int_{0}^{1} z(x, t) \mathcal{L}_{j}(x) d x . \\
& =-\Lambda z(1, t)+(-1)^{k} \Lambda z(0, t)-F\left(Z_{N}(t)\right)_{k} \\
& +\sum_{j=0}^{\max [0, k-1]} \ell_{k j} \Lambda\left(Z_{N}(t)\right)_{j} d x .
\end{aligned}
$$

Consequently, using the notations recently introduced, we have

$$
\begin{aligned}
\frac{d}{d t} Z_{N}(t)=\left(L_{N}(\Lambda)-\right. & \left.\mathcal{F}_{N}\right) Z_{N}(t)-\mathbb{1}_{N}(\Lambda) z(1, t) \\
& +\mathbb{1}_{N}(\Lambda)^{*} z(0, t)
\end{aligned}
$$

Injecting the boundary condition $z(0, t)=C X(t)+$ $D z(1, t)$ in the previous expression allows to conclude the proof.

To lead stability analysis for system (1), we will need the Bessel inequality comparing an $L^{2}(0,1)$ scalar product with the corresponding finite dimensional approximation.

Remark 3: Using Legendre polynomials allows to keep the interconnection between all components of $\dot{Z}_{N}(t)$ since the matrix $L_{N}(\Lambda)$ is lower triangular. Thus, we conserve the hierarchy of this stability study. We can use other basis of polynomials for this stability study, except trigonometric functions which lead to a different expression of $\dot{Z}_{N}(t)$ without any links between its components.

\section{A. Bessel inequality}

This paragraph recalls the Bessel-Legendre inequality lemma. This lemma is based on the error between a vector and its projection. Taking advantage of the positivity of this error, it allows to limit the vector by a lower or upper bound.

Lemma 1: Let $z \in L^{2}\left(0,1 ; \mathbb{R}^{m}\right)$ and $R \in \mathbb{S}_{+}^{m}$. The following integral inequality holds for all $N \in \mathbb{N}$ :

$$
\int_{0}^{1} z^{\top}(x) R z(x) d x \geq Z_{N}^{\top} R^{N} Z_{N}
$$

with

$$
R^{N}=\operatorname{diag}(R, 3 R, \ldots,(2 N+1) R),
$$

Proof: The proof is easily led by considering the difference between the variable $z$ and its projections over the $N+1$ first Legendre polynomials. Indeed, denoting

$$
y_{N}(x)=z(x)-\sum_{k=0}^{N} \frac{\mathcal{L}_{k}(x)}{\left\|\mathcal{L}_{k}\right\|^{2}} \int_{0}^{1} z(\xi) \mathcal{L}_{k}(\xi) d \xi
$$


the orthogonality of the Legendre polynomials and the Bessel inequality leads to (10) from the positive definiteness and the expansion of $\int_{0}^{1} y_{N}^{\top}(x) R y_{N}(x) d x$, as in e.g. $[15]$.

\section{Stability ANALYSis}

In this section, we address the stability analysis of system (1) using the Lyapunov functional (III), Property 1 and Lemma 1 . Before giving the stability theorem of system (1), we recall that the matrices $L_{N}(\Lambda), \mathbb{1}_{N}(\Lambda)$, $\mathcal{F}_{N}$ and $\mathbb{1}_{N}^{*}(\Lambda)$ are defined in (8), the matrix $R^{N}$ is given by (10) and define the following $\mathbb{R}^{m(N+1), m(N+1)}$ matrices

$$
\begin{aligned}
S^{N} & =\operatorname{diag}(S, 3 S, \ldots,(2 N+1) S) \\
M^{N} & =\operatorname{diag}(M, 3 M, \ldots,(2 N+1) M) \\
\mathcal{I}^{N} & =\operatorname{diag}\left(I_{m}, 3 I_{m}, \ldots,(2 N+1) I_{m}\right) .
\end{aligned}
$$

Theorem 1: Consider system (1) with a given transport speed matrix $\Lambda \succ 0$. If there exists an integer $N>0$ such that there exists

- $P \in \mathbb{S}_{+}^{n}, Q_{N} \in \mathbb{R}^{n,(N+1) m}$ and $T_{N} \in \mathbb{S}^{(N+1) m}$,

- $M, S$ and $R \in \mathbb{S}_{+}^{m}$,

satisfying the following LMIs

$$
\begin{gathered}
\Phi_{N}(\Lambda)=\left[\begin{array}{cc}
P & Q_{N} \\
Q_{N}^{\top} & T_{N}+S^{N}
\end{array}\right] \succ 0, \\
\Psi_{N}(\Lambda)=\left[\begin{array}{ccc}
\Psi_{11} & \Psi_{12} & \Psi_{13} \\
* & \Psi_{22} & \Psi_{23} \\
* & * & \Psi_{33}
\end{array}\right] \prec 0, \\
\Gamma_{1}(\Lambda)=\Lambda R+\operatorname{He}\left(F^{\top}(S+R)\right) \succ M, \\
\Gamma_{2}(\Lambda)=\Lambda R+\operatorname{He}\left(F^{\top} S\right) \succ M,
\end{gathered}
$$

where

$$
\begin{aligned}
& \Psi_{11}=\operatorname{He}\left(P A+Q_{N} \mathbb{1}_{N}^{*}(\Lambda) C\right)+C^{T} \Lambda(S+R) C \\
& \Psi_{12}=P B+Q_{N}\left(\mathbb{1}_{N}^{*}(\Lambda) D-\mathbb{1}_{N}(\Lambda)\right)+C^{\top} \Lambda(S+R) D \\
& \Psi_{13}=A^{\top} Q_{N}+C^{\top} \mathbb{1}_{N}^{* \top}(\Lambda) T_{N}+Q_{N}\left(L_{N}(\Lambda)-\mathcal{F}_{N}\right) \\
& \Psi_{22}=-\Lambda S+D^{\top} \Lambda(S+R) D \\
& \Psi_{23}=B^{\top} Q_{N}+\left(\mathbb{1}_{N}^{*}(\Lambda) D-\mathbb{1}_{N}(\Lambda)\right)^{\top} T_{N}, \\
& \Psi_{33}=\operatorname{He}\left(T_{N}\left(L_{N}(\Lambda)-\mathcal{F}_{N}\right)\right)-M^{N},
\end{aligned}
$$

then system (1) is exponentially stable.

Proof: Our objective to prove this stability result is to show that the Lyapunov functional $V_{N}$ given in (2) satisfies the inequalities (6) and (7) for positive scalars $\varepsilon_{1}, \varepsilon_{2}$ and $\varepsilon_{3}$. The proof falls then into three steps.

For simplicity, the expression $V_{N}(t)=V_{N}(X(t), z(t))$ will be used hereafter.

Step 1: Since $S \succ 0$ and $\Phi_{N} \succ 0$, there exists a sufficiently small $\varepsilon_{1}>0$ such that $S \succ \varepsilon_{1} I_{m}$ and

$$
\Phi_{N}=\left[\begin{array}{cc}
P & Q_{N} \\
* & T_{N}+S^{N}
\end{array}\right] \succ \varepsilon_{1}\left[\begin{array}{cc}
I_{n} & 0 \\
* & \mathcal{I}^{N}
\end{array}\right]
$$

Moreover, $V_{N}$ defined by (2) satisfies, for all $t \geq 0$,

$$
\begin{aligned}
V_{N}(t) & \geq\left[\begin{array}{c}
X(t) \\
Z_{N}(t)
\end{array}\right]^{\top} \Phi_{N}(\Lambda)\left[\begin{array}{c}
X(t) \\
Z_{N}(t)
\end{array}\right] \\
& -Z_{N}^{\top}(t) S^{N} Z_{N}(t)+\int_{0}^{1^{\top}} z^{\top}(x, t) S z(x, t) d x .
\end{aligned}
$$

Then, replacing $\Phi_{N}$ by its lower bound and introducing $\varepsilon_{1}$ in the last term, we have

$$
\begin{aligned}
V_{N}(t) \geq & \varepsilon_{1}|X(t)|_{n}^{2}+\varepsilon_{1} \int_{0}^{1} z^{\top}(x, t) z(x, t) d x \\
& -Z_{N}^{\top}(t)\left(S^{N}-\varepsilon_{1} \mathcal{I}^{N}\right) Z_{N}(t) \\
& +\int_{0}^{1} z^{\top}(x, t)\left(S-\varepsilon_{1} I_{m}\right) z(x, t) d x
\end{aligned}
$$

Since $S-\varepsilon_{1} I_{m} \succ 0$, applying Lemma 1 we obtain that the sum of the two last terms is positive. we then have proved that $\exists \varepsilon_{1}>0$ such that

$$
V_{N}(t) \geq \varepsilon_{1}\left(|X(t)|_{n}^{2}+\|z(t)\|_{L^{2}\left(0,1 ; \mathbb{R}^{m}\right)}^{2}\right) \geq \varepsilon_{1} E(t) .
$$

Step 2: There exists a sufficiently large scalar $\beta>0$ that allows

$$
\left[\begin{array}{cc}
P & Q_{N} \\
Q_{N}^{\top} & T_{N}
\end{array}\right] \preceq \beta\left[\begin{array}{cc}
I_{n} & 0 \\
* & \mathcal{I}^{N}
\end{array}\right]
$$

such that since $S \succ 0$ and $R \succ 0$, we get

$$
\begin{aligned}
& V_{N}(t) \leq \beta|X(t)|_{n}^{2}+\beta Z_{N}^{\top}(t) \mathcal{I}^{N} Z_{N}(t) \\
& +\int_{0}^{1} z^{\top}(x, t)(S+(1-x) R) z(x, t) d x .
\end{aligned}
$$

Taking the upper bound of $(1-x)$, we get

$$
\begin{aligned}
V_{N}(t) \leq \beta & |X(t)|_{n}^{2}+\beta Z_{N}^{\top}(t) \mathcal{I}^{N} Z_{N}(t) \\
& +\int_{0}^{1} z^{\top}(x, t)(S+R) z(x, t) d x .
\end{aligned}
$$

Applying Lemma 1 to the second term of the right-hand side gives

$$
\begin{aligned}
V_{N}(t) & \leq \beta|X(t)|_{n}^{2}+\int_{0}^{1} z^{\top}(x, t)\left(\beta I_{m}+S+R\right) z(x, t) d x \\
& \leq \beta|X(t)|_{n}^{2}+\varepsilon_{2}\|z\|_{L^{2}\left(0,1 ; \mathbb{R}^{m}\right)}^{2} \leq \varepsilon_{2} E(t),
\end{aligned}
$$

where $\varepsilon_{2}=\beta+\lambda_{\max }(S)+\lambda_{\max }(R)$. Therefore, the proof of $(6)$ is complete.

Step 3: To simplify the computations and the notations hereafter, we divide the Lyapunov functional (2) into two terms:

$$
\begin{aligned}
V_{N, 1}(t) & =\left[\begin{array}{c}
X(t) \\
Z_{N}(t)
\end{array}\right]^{\top}\left[\begin{array}{cc}
P & Q_{N} \\
Q_{N}^{\top} & T_{N}
\end{array}\right]\left[\begin{array}{c}
X(t) \\
Z_{N}(t)
\end{array}\right], \\
V_{N, 2}(t) & =\int_{0}^{1} z^{\top}(x, t)(S+(1-x) R) z(x, t) d x,
\end{aligned}
$$

and let us define the finite dimensional augmented state vector, of size $n+(N+2) m$ given by

$$
\xi_{N}(t)=\left[\begin{array}{lll}
X^{\top}(t) & z^{\top}(1, t) & Z_{N}^{\top}(t)
\end{array}\right]^{\top} .
$$

Computing the derivative of Lyapunov functional $V_{N}(t)$ in order to determine the positive scalar $\varepsilon_{3}$ satisfying equation (7), yields

$\dot{V}_{N, 1}(t)=\xi_{N}^{\top}(t)\left[\begin{array}{ccc}\psi_{1} & \psi_{2} & \Psi_{13} \\ * & 0 & \Psi_{23} \\ * & * & \operatorname{He}\left(T_{N}\left(L_{N}(\Lambda)-\mathcal{F}_{N}\right)\right)\end{array}\right] \xi_{N}(t)$, 
where $\Psi_{13}$ and $\Psi_{23}$ are defined in Theorem 1, and

$$
\begin{aligned}
& \psi_{1}=\operatorname{He}\left(P A+Q_{N} \mathbb{1}_{N}^{*}(\Lambda) C\right), \\
& \psi_{2}=P B+Q_{N}\left(\mathbb{1}_{N}^{*} D-\mathbb{1}_{N}(\Lambda)\right) .
\end{aligned}
$$

Now, using the transport equation in (1), we have

$$
\begin{gathered}
\dot{V}_{N, 2}(t)=\int_{0}^{1} \partial_{t}\left(z^{\top}(x, t)(S+(1-x) R) z(x, t)\right) d x \\
=-2 \int_{0}^{1}\left(\Lambda \partial_{x} z(x, t)\right)^{\top}(S+(1-x) R) z(x, t) \\
\quad+(F z(x, t))^{\top}(S+(1-x) R) z(x, t) d x \\
=-\left[z^{\top}(x, t) \Lambda(S+(1-x) R) z(x, t)\right]_{0}^{1} \\
-\int_{0}^{1} z^{\top}(x, t)\left[\operatorname{He}\left(F^{\top}(S+(1-x) R)\right)+\Lambda R\right] z(x, t) d x
\end{gathered}
$$

Merging the expressions of $\dot{V}_{N, 1}$ and $\dot{V}_{N, 2}$, we obtain

$$
\begin{aligned}
& \dot{V}_{N}(t)=\xi_{N}^{\top}(t)\left[\begin{array}{ccc}
\psi_{1} & \psi_{2} & \Psi_{13} \\
* & 0 & \Psi_{23} \\
* & * & \operatorname{He}\left(T_{N}\left(L_{N}(\Lambda)-\mathcal{F}_{N}\right)\right)
\end{array}\right] \xi_{N}(t) \\
& +z^{\top}(0, t) \Lambda(S+R) z(0, t)-z^{\top}(1, t) \Lambda S z(1, t) \\
& -\int_{0}^{1} z^{\top}(x, t)\left[\operatorname{He}\left(F^{\top}(S+(1-x) R)\right)+\Lambda R\right] z(x, t) d x .
\end{aligned}
$$

To take the upper bound of the integral term, we consider that their exists a positive matrix $M$ such that

$$
\begin{aligned}
& \Lambda R+\operatorname{He}\left(F^{\top}(S+R)\right) \succ M, \\
& \Lambda R+\operatorname{He}\left(F^{\top} S\right) \succ M .
\end{aligned}
$$

we have thus, by convexity

$$
\Lambda R+\operatorname{He}\left(F^{\top}(S+(1-x) R)\right) \succ M \quad \forall x \in[0,1],
$$

Then, we can write

$$
\begin{aligned}
& \dot{V}_{N}(t) \leq \xi_{N}^{\top}(t)\left[\begin{array}{ccc}
\psi_{1} & \psi_{2} & \Psi_{13} \\
* & 0 & \Psi_{23} \\
* & * & \operatorname{He}\left(T_{N}\left(L_{N}(\Lambda)-\mathcal{F}_{N}\right)\right)
\end{array}\right] \xi_{N}(t) \\
& +z^{\top}(0, t) \Lambda(S+R) z(0, t)-z^{\top}(1, t) \Lambda S z(1, t) \\
& -\int_{0}^{1} z^{\top}(x, t) M z(x, t) d x .
\end{aligned}
$$

From Lemma 1 , since $M>0$ we have

$$
\int_{0}^{1} z^{\top}(x, t) M z(x, t) d x \geq Z_{N}^{\top}(t) M Z_{N}(t)
$$

Injecting the boundary condition $z(0, t)=C X(t)+$ $D z(1, t)$ and using the definition of the matrix $\Psi_{N}(\Lambda)$ in (13), the following estimate of $\dot{V}_{N}$ is obtained:

$$
\dot{V}_{N}(t) \leq \xi_{N}^{\top}(t) \Psi_{N}(\Lambda) \xi_{N}(t)
$$

Finally, the LMI (13) ensures that there exists a sufficiently small $\varepsilon_{3}>0$ such that

$$
M \succ \varepsilon_{3} I_{m}, \quad \Psi_{N}(\Lambda) \prec-\varepsilon_{3}\left[\begin{array}{ccc}
I_{n} & 0 & 0 \\
0 & 0 & 0 \\
0 & 0 & \mathcal{I}^{N}
\end{array}\right] .
$$

Hence, using the definition (15) of $\xi_{N}(t)$ and these two LMIs in (16) yields

$$
\begin{aligned}
\dot{V}_{N}(t) & \leq-\varepsilon_{3}\left(|X(t)|_{n}^{2}+\int_{0}^{1}|z(x, t)|^{2} d x\right) \\
& +Z_{N}^{\top}(t)\left(M^{N}-\varepsilon_{3} \mathcal{I}^{N}\right) Z_{N}(t) \\
& -\int_{0}^{1} z^{\top}(x, t)\left(M-\varepsilon_{3} I_{m}\right) z(x, t) d x .
\end{aligned}
$$

Since $M-\varepsilon_{3} I_{m} \succ 0$, Lemma 1 can be applied and gives

$$
\begin{aligned}
-\int_{0}^{1} z^{\top}(x, t) & \left(M-\varepsilon_{3} I_{m}\right) z(x, t) d x \\
& \leq-Z_{N}^{\top}(t)\left(M^{N}-\varepsilon_{3} \mathcal{I}^{N}\right) Z_{N}(t) .
\end{aligned}
$$

Thus, the Lyapunov functional $V_{N}$ satisfies, for all $t>0$

$$
\dot{V}_{N}(t) \leq-\varepsilon_{3} E(t)
$$

We can therefore conclude on the asymptotic stability of system (1).

\section{Numerical Examples}

To test our stability results, we consider system (1) with following matrices

$$
\begin{array}{ccc}
A= & {\left[\begin{array}{cc}
-2 & 0 \\
0 & -0.9
\end{array}\right],} & B=\left[\begin{array}{cccc}
-1 & 0 & -1 & 0 \\
-1 & -1 & -1 & -1
\end{array}\right], \\
C= & {\left[\begin{array}{cc}
0.05 & 0 \\
0 & 0.05 \\
0 & 0 \\
0 & 0
\end{array}\right], \quad D=\left[\begin{array}{cccc}
0 & 0 & 0 & 0 \\
0 & 0 & 0 & 0 \\
19 & 0 & 0 & 0 \\
0 & 19 & 0 & 0
\end{array}\right],}
\end{array}
$$

and the transport speed matrix $\Lambda$ is given by

$$
\Lambda=\left[\begin{array}{cc}
\rho_{1} I_{2} & 0 \\
0 & \rho_{2} I_{2}
\end{array}\right] .
$$

To observe the effect of the potential term on stability of the system, we compute the minimum transport speed value for several values of $F$. We consider the following matrix of the potential term $F=\sigma\left[\begin{array}{ll}0_{2} & I_{2} \\ I_{2} & 0_{2}\end{array}\right]$. This form of the matrix $F$ allows to inject the second half of the state $z(x, t)$ in the first equation, and the first one in the second equation.

Taking $\rho_{1}=\rho_{2}=\rho$, Figure 2 gives the minimum transport speed $\rho$ for which the system remains stable.

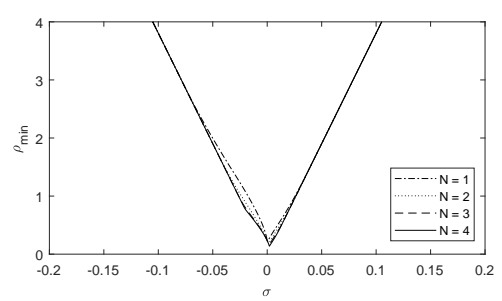

Fig. 2: Minimum transport speed depends on $\sigma$ for several values of $N$

We note in Figure 2 at $\sigma=0$, corresponds to system 1 without the potential term $F$, that we get the example studied in [9] and [14]. For $N=4$, we get the minimum transport speed value $\rho_{\min }=0.2327$ (maximum delay 
value $h=4.2973$ ) for which the system remains stable. This maximum delay gets near the value found in [9] by increasing the order $N$ of Legendre polynomials.

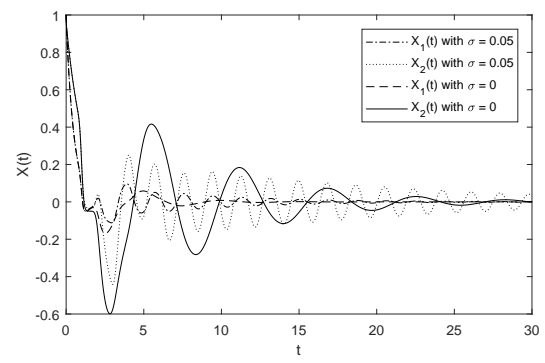

Fig. 3: Simulation of $X(t)$ of the coupled system with $\rho_{1}=\rho_{2}=1$.

We remark in Figure 3 that the response $X(t)$ of the coupled system is more oscillating with a determined potential term in the transport $\operatorname{PDE}\left(X(t)=\left[\begin{array}{l}X_{1}(t) \\ X_{2}(t)\end{array}\right]\right.$ with $\sigma=0.05)$ than that obtained without potential $\left(X(t)=\left[\begin{array}{l}X_{1}(t) \\ X_{2}(t)\end{array}\right]\right.$ with $\left.\sigma=0\right)$. Therefore, adding a potential term to the transport PDE of the coupled system (1) may introduce more oscillations to the system.

To have more stability result of system (1), we consider the same example with $\rho_{1}=2 \rho_{2}=2 \rho$. This form allows us to avoid the result of Remark 1 which consists in reducing system (1) on a simple coupled ODE-PDE system without the potential term.

In Figure 4, we observe that the stability conditions form a hierarchy of LMI, i.e. if the system is stable at the order $N$ of Legendre polynomials, it remains stable for all higher orders greater than $N$.

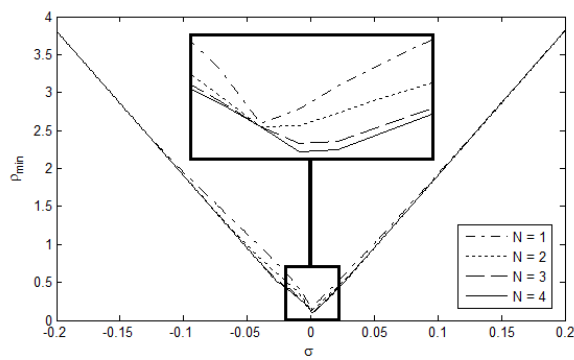

Fig. 4: Minimum transport speed depends on $\sigma$ for several values of $N$

We also note in Figure 4 that we can expand the interval of stability of this system by choosing an appropriate value of $F$ (for $N=4$, we have $\rho_{\text {min }}=0.0976$ for $F=0.001\left[\begin{array}{ll}0_{2} & I_{2} \\ I_{2} & 0_{2}\end{array}\right]$ instead of $\rho_{\min }=0.1256$ for $F=$ $0_{4}$ ). Thus, adding the potential term to the transport equation with an appropriate value may broaden the interval of stability of the coupled system (1).

\section{Conclusion}

This work provides a novel approach for stability of a system coupling an ODE to a hyperbolic transport PDE with potential term, using Lyapunov methodology based on Bessel inequality and Legendre polynomials. Indeed, it shows how the potential term in the transport PDE may improve the stability result of the coupled system depending on its sign. In other words, interconnecting PDEs each other only in the undifferentiated terms may expand the stability interval of the coupled ODE-PDE system. Moreover, the set of stability conditions forms a hierarchy of LMI indexed by the polynomial degree $\mathrm{N}$, and increasing this degree may only improve the stability results. For future research, the potential term of the coupled ODE-PDE system can depend on time and/or spatial variable $x$.

\section{REFERENCES}

[1] G. Bastin and J-M. Coron. Stability and boundary stabilization of 1-d hyperbolic systems. Springer, 2016.

[2] L. Baudouin, A. Seuret, and M. Safi. Stability analysis of a system coupled to a transport equation using integral inequalities. In IFAC Conference on Control of Systems Governed by Partial Differential Equations (CPDE'16), Bertinoro, Italy, 2016.

[3] F. Castillo, E. Witrant, C. Prieur, V. Talon, and L. Dugard. Fresh air fraction control in engines using dynamic boundary stabilization of LPV hyperbolic systems. IEEE Transactions on Control Systems Technology, 23(3):963-974, 2015.

[4] G-M. Coclite, M. Garavello, and B. Piccoli. Traffic flow on a road network. SIAM Journal on Mathematical Analysis, 36(6):1862-1886, 2005.

[5] J-M Coron. Control and nonlinearity. Number 136. American Mathematical Soc., 2007.

[6] R. Courant and D. Hilbert. Methods of mathematical physics. Interscience publishers, New York, 1, 1953.

[7] F. Di Meglio, R. Vazquez, and M. Krstic. Stabilization of a system of $n+1$ coupled first-order hyperbolic linear PDEs with a single boundary input. IEEE Transactions on Automatic Control, 58(12):3097-3111, Dec 2013.

[8] L. C. Evans. Partial differential equations. Graduate Studies in Mathematics, 19, 1998.

[9] K. Gu, J. Chen, and V. L. Kharitonov. Stability of time-delay systems. Springer Science \& Business Media, 2003.

[10] M. Gugat, M. Dick, and G. Leugering. Gas flow in fan-shaped networks: Classical solutions and feedback stabilization. SIAM Journal on Control and Optimization, 49(5):2101-2117, 2011.

[11] D. Jacquet, M. Krstic, and C. Canudas de Wit. Optimal control of scalar one-dimensional conservation laws. In American Control Conference, 2006, 2006.

[12] M Krstic. Delay compensation for nonlinear, adaptive, and PDE systems. Springer, 2009.

[13] M. Krstic and A. Smyshlyaev. Backstepping boundary control for first-order hyperbolic PDEs and application to systems with actuator and sensor delays. Systems \& Control Letters, 57(9):750 - 758, 2008.

[14] M. Safi, L. Baudouin, and A. Seuret. Refined exponential stability analysis of a coupled system. IFAC Conference, Toulouse, France, 2017.

[15] M. Safi, L. Baudouin, and A. Seuret. Tractable sufficient stability conditions for a system coupling linear transport and differential equations. Systems \& Control Letters, 110:1 - 8, 2017.

[16] A. Seuret and F. Gouaisbaut. Hierarchy of LMI conditions for the stability analysis of time-delay systems. Systems 83 Control Letters, 81:1-7, 2015.

[17] Y. Tang, C. Prieur, and A. Girard. Stability analysis of a singularly perturbed coupled ODE-PDE system. In Conference on Decision and Control, Osaka, Japan, 2015.

[18] E. Witrant, E. Joffrin, S. Brémond, G. Giruzzi, D. Mazon, O. Barana, and P. Moreau. A control-oriented model of the current profile in tokamak plasma. Plasma Physics and Controlled Fusion, 49(7):1075, 2007. 